\title{
Preprocessing by means of Subspace Projections for Continuous Cerebral Autoregulation Assessment Using NIRS*
}

\author{
A. Caicedo, Member, IEEE, G. Naulaers, S. Van Huffel Fellow, IEEE
}

\begin{abstract}
Cerebral Autoregulation (CA) refers to the capability of the brain to maintain a more or less stable cerebral blood flow (CBF), despite the changes in blood perfusion. Monitoring this mechanism is of vital importance, especially in neonates, in order to prevent damage due to ischemia or hemorrhage. In clinical practice near-infrared spectroscopy (NIRS) measurements are used as a surrogate measurement for CBF. However, NIRS signals are highly dependent on the variations in arterial oxygen saturation $\left(\mathrm{SaO}_{2}\right)$. Therefore, only segments with relatively constant $\mathrm{SaO}_{2}$ are used for $\mathrm{CA}$ assessment; which limits the possibilities of the use of NIRS for online monitoring. In this paper we propose the use of subspace projections to subtract the influence of $\mathrm{SaO}_{2}$ from NIRS measurements. Since this approach will be used in an online monitoring system, this preprocessing is carried out in a window-by-window framework. However, the use of subspace projections in consecutive segments produces discontinuities; we propose a methodology to reduce these effects. Obtained results indicate that the proposed method reduces the effect of discontinuities between consecutive segments. In addition, this methodology is able to subtract the influence of $\mathrm{SaO}_{2}$ from NIRS measurements. This approach facilitates the introduction of NIRS for online CA assessment.
\end{abstract}

\section{INTRODUCTION}

Cerebral autoregulation (CA) is the capability of the brain to maintain a more or less constant cerebral blood flow (CBF) despite changes in mean arterial blood pressure (MABP). Cerebral autoregulation was first described in [1], where experimental measures showed that CBF was kept more or less constant along a large range of MABP values. In the literature it is mentioned that this mechanism may be impaired, or immature, in premature neonates [2], [3]. Impaired CA may lead to cerebral damage due to ischemia, in case of low MABP values, or hemorrhage, in case of high

*Research supported by: Research Council KUL: GOA MaNet, PFV/10/002 (OPTEC), IDO 08/013 Autism, several PhD/postdoc \& fellow grants; Flemish Government: FWO: $\mathrm{PhD} /$ postdoc grants, projects: G.0427.10N (Integrated EEG-fMRI), G.0108.11 (Compressed Sensing) G.0869.12N (Tumor imaging) G.0A5513N (Deep brain stimulation) IWT: TBM070713-Accelero, TBM080658-MRI (EEG-fMRI), TBM110697NeoGuard, PhD Grants; iMinds 2012, Flanders Care: Demonstratieproject Tele-Rehab III (2012-2014). Belgian Federal Science Policy Office: IUAP P719/ (DYSCO, 'Dynamical systems, control and optimization', 20122017); ESA AO-PGPF-01, PRODEX (CardioControl) C4000103224. EU: RECAP 209G within INTERREG IVB NWE programme, EU HIP Trial FP7-HEALTH/ 2007-2013 (n 260777), EU ITN Transact 2012.

A. C. is with the Electronic Engineering Department, ESAT/SCD SISTA, KU Leuven, Leuven, Belgium. He is also with iMinds Future Health Department, Katholieke Universiteit Leuven, Leuven, Belgium (phone: +32 16 321067; e-mail: acaicedo@esat.kuleuven.be).

G. N. is with the University Hospitals Leuven, Gasthuisberg, KU Leuven, Leuven, Belgium (e-mail: gunnar.naulaers@uzleuven.be)

$\mathrm{S}$. VH. is with the Electronic Engineering Department, ESAT/SCD SISTA, Katholieke Universiteit Leuven, Leuven, Belgium. She is also with iMinds Future Health Department, KU Leuven, Leuven, Belgium (e-mail: $\underline{\text { sabine.vanhuffel@esat.kuleuven.be). }}$
MABP values. Therefore, it is of vital importance to monitor $\mathrm{CA}$ in this population. Even though, strong evidence that links CA impairment with cerebral lesion is still scarce.

Assessment of $\mathrm{CA}$ is based on the quantification of the strength in the relationship between MABP and CBF. However, measurements of $\mathrm{CBF}$ are difficult to obtain continuously in a clinical environment. For this reason surrogate measures are used in practice. Near-infrared Spectroscopy (NIRS) is a technology that measures the variations in oxy- and deoxy-haemoglobin. From these measurements the tissue oxygenation index (TOI), defined as the percentage of oxygen available in the tissue, and the difference in haemoglobin concentration $(\Delta \mathrm{HbD})$ can be computed. The expressions to calculate these values are shown in (1).

$$
\begin{aligned}
& T O I=\frac{k H b O_{2}}{k H b O_{2}+k H H b} \\
& \Delta H b D=\Delta H b O_{2}-\Delta H H b
\end{aligned}
$$

Where $\mathrm{HbO}_{2}$ and $\mathrm{HHb}$ represent oxy- and deoxyhemoglobin concentrations, respectively, $k$ is a contants, $\triangle \mathrm{HbO}_{2}$ and $\mathrm{\triangle HHb}$ represent the changes in oxy- and deoxyhaemoglobin concentrations, respectively. In [4] it was shown that TOI measurements are more appropriate for cerebral autoregulation assessment, since they are less affected by movement artifacts. In [5] the authors described that, under a constant brain metabolic demand and constant arterial oxygen saturation $\left(\mathrm{SaO}_{2}\right)$, changes in TOI and $\mathrm{HbD}$ represent changes in $\mathrm{CBF}$. Several studies have been presented in the literature that evaluates the use of NIRS as a promising tool for cerebral haemodynamics monitoring, [6], [7], and [8].

In premature neonates it can be assumed that the cerebral metabolic demand is constant during the first days of life. However, changes in $\mathrm{SaO}_{2}$ influence directly changes in TOI or $\mathrm{HbD}$. For this reason, only segments with stable $\mathrm{SaO}_{2}$ are used for CA assessment. This limits the possibilities of NIRS to be used for online CA monitoring. This limitation can be mitigated by an appropriated preprocessing algorithm, which eliminates the influence of $\mathrm{SaO}_{2}$ on $\mathrm{TOI} / \mathrm{HbD}$. In a previous study we presented an algorithm, based on the use of orthogonal and oblique subspace projection techniques (OrSP), to decompose a target signal in a set of signals related to a given basis. In this study we exploit the use of this algorithm as a preprocessing step for CA assessment [9].

However, OrSP is a frame based approach, and the computation of the projection matrices is done using the available data in the frame. Therefore, projections in consecutive frames may present high discontinuities, mainly due to border problems. Moreover, the length of the frame is limited by the hardware characteristics; a frame longer than 
3000 data points will require a high computing power to be processed, which is not suitable for online monitoring. In order to cope with this problem, we propose to reduce the influence of continuous frames by including a prediction horizon and reducing the difference in the mean value between the projections in the actual and previous frame.

\section{ORThOGONAL AND OBLIQUe PROJECTIONS}

A projection is a linear transformation that maps any vector in the column space defined by some basis, from which the projection operator was derived. There are two kinds of projection matrices, orthogonal and oblique projections. Orthogonal projections project orthogonally any vector onto the column space defined by a basis; while oblique projections project the vector onto the column space defined by the basis along the direction of a reference subspace. The orthogonal projection can be seen as a special case of an oblique projection, where the reference subspace is orthogonal to the subspace defined by the given basis. In [10] the use of orthogonal and oblique subspace projections in signal processing applications is presented. A more theoretical background about projection matrices can be found in [11].

\section{A. Projection Matrices}

Let the matrix $\boldsymbol{X}$ of size $m x n$ be composed of the vectors $\left\{x_{1} x_{2}, \ldots x_{n}\right\}$, with $x_{i} \in \mathfrak{R}^{m}$, in its columns. Assuming that $\boldsymbol{X}$ is full rank (rank $=n$, with $m>n$ ) the vectors $\left\{x_{l}, \ldots, x_{n}\right\}$ form a basis for the column space of $\boldsymbol{X}$. The orthogonal projection matrix can be calculated as $\boldsymbol{P}=\boldsymbol{X}\left(\boldsymbol{X}^{T} \boldsymbol{X}\right)^{-1} \boldsymbol{X}^{T}$. Now let the matrix $Z$ of size $m x r$ composed of the vectors $\left\{z_{1} z_{2}, \ldots z_{r}\right\}$, with $\mathrm{Z}_{i} \in \mathfrak{S}^{m}$, in its columns, represent a reference subspace. The oblique projection onto the column space of $\mathrm{X}$ along the column space of $\mathrm{Z}, P_{X \mid Z}$, is given by (2).

$$
P_{X \mid Z}=X\left(X^{T} Q_{Z} X\right)^{-1} X^{T} Q_{Z}
$$

Where $Q_{Z}=I-P_{Z}$ represents the orthogonal projection onto the null space of $Z^{T}, N\left(Z^{T}\right)$, with $\mathrm{P}_{\mathrm{z}}$ the orthogonal projection onto the column space of $\mathrm{Z}, C(Z)$.

\section{B. Consecutive Projection Matrices}

The calculation of a projection matrix is a $O\left(N^{3}\right)$ operation; therefore, for large $N$ a faster approach will be to segment the data into smaller windows, and perform the calculation in each individual window. In addition this segmentation also reduces the effect of nonstationarities. Present in each frame which implies that the projection matrices are likely to change from frame to frame. Hence, the projections on consecutive frames might present high discontinuities in the borders. Therefore, the use of this approach, without correction, in monitoring applications is not feasible.

There are several sources for the discontinuities between consecutive frames, as given below:

- In the calculation of projection matrices the data should be centered, differences in the centering between consecutive frames produce differences when the data are projected. This bias effect is mild and can be corrected by overlapping consecutive frames and computing the bias between the projections in the previous and the actual frame.

- Border distortions also introduce an error in the projections. This effect can be reduced by including a future horizon in the basis. Consider the observation matrix $\mathbf{X}$ defined before, and a future horizon $\mathbf{X}_{\mathbf{f}}$ of size $p x n$, the projection matrix can be calculated using the extended matrix $\boldsymbol{X}_{\boldsymbol{E}}=\left[\boldsymbol{X} ; \boldsymbol{X}_{\boldsymbol{f}}\right]$. By projecting the data onto this new subspace and removing the last $p$ data points, the border problem is reduced.

- Consecutive projectors in time might represent the same subspace, but the projections can present a change in phase of $180^{\circ}$. This problem can be addressed by overlapping consecutive segments and checking the sign of the correlation between the projections in the overlapped segment. This correlation should be positive; in case of negative correlation the projection in the new frame should be inverted. It is important to note that the longer the overlap the more reliable the correlation estimator.

- $\quad$ Finally, changes in the dynamics of the system, due to non-stationarities, affect the column space defined by the basis. This problem is not completely addressed in this study. However, by the inclusion of a future horizon and an overlapping between consecutive frames these changes are smoothed.

\section{METHODS}

Recordings from 20 infants from the University Hospital Leuven (Belgium), with a gestational age of $28.4 \pm 3.5$ weeks and a birth weight of $1113 \pm 499$ grams were used in this study. In all infants the peripheral oxygen saturation $\left(\mathrm{SaO}_{2}\right)$ was measured continuously by pulse oximetry, and MABP by an indwelling arterial catheter. With NIRS, the $\triangle \mathrm{HbD}$ and the tissue oxygenation index (TOI) were continuously and non-invasively recorded using the NIRO 300 (Hamamatsu). MABP, $\mathrm{SaO}_{2}$ and NIRS signals were simultaneously measured during the first three days of life and downsampled at $0.333 \mathrm{~Hz}$. The total length of the recordings was 6-9 hours. The recordings were segmented in epochs without movement

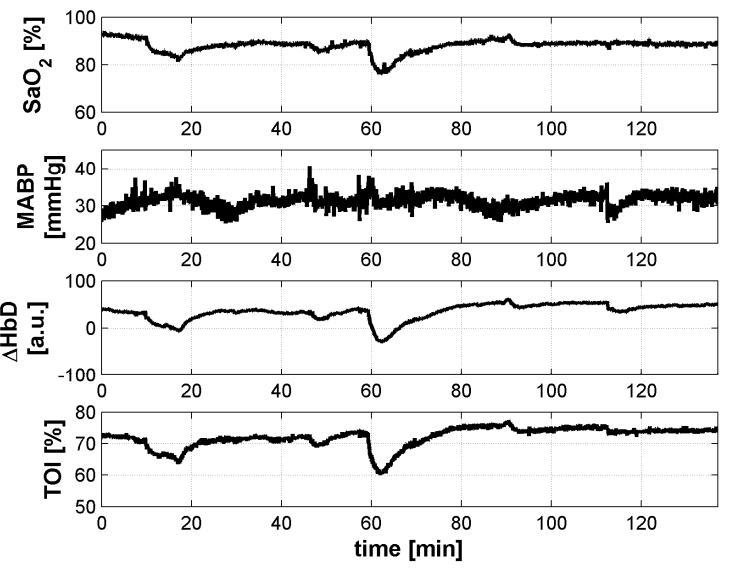

Figure 1. Representative recording. From top to bottom, $\mathrm{SaO}_{2}$, $M A B P, \triangle H b D$ in arbitrary units, and TOI. A strong relation between $\mathrm{SaO}_{2}$ variations and TOI/ $\triangle \mathrm{HbD}$ is shown. 
artefacts. These epochs were used for further processing.

In figure 1 a representative recording, where variations in $\mathrm{SaO}_{2}$ are noticeable, is shown. This recording belongs to a premature infant during his first day of life and is 137 minutes long. This recording will be used in order to illustrate the performance of the method presented in this paper.

Two different analyses were performed. In the first analysis the complete recording was used in order to illustrate how the orthogonal projection algorithms can be applied. In the second analysis a sliding window was used to simulate the effect of continuous monitoring. For the first analysis the subspaces for the MABP and $\mathrm{SaO}_{2}$ were defined as follows:

- A discrete wavelet transformation, of 4 levels using Daubechies 4 as mother wavelet, was applied to the raw data [12].

- A block Hankel matrix is created using the results obtained from the wavelet transform, using a delay up to 10 samples.

- The projection matrices onto MABP along $\mathrm{SaO}_{2}$, and onto $\mathrm{SaO}_{2}$ along MABP were computed as indicated in (2).

- $\quad$ The TOI was projected onto these subspaces.

For the second analysis, the signal was segmented in consecutive windows of 400 samples (20 minutes) with a future horizon of 40 samples (2 minutes), an overlapping of 18 minutes between consecutive frames was used in order to produce a new projection every 2 minutes. For each frame the projection matrices were calculated following the same procedure explained before. Correction for the bias was performed as explained before.

Furthermore, correlation and coherence values between TOI, before and after preprocessing, and $\mathrm{SaO}_{2}$ were computed in order to evaluate the overall performance of the method. Coherence coefficients were averaged in the frequency range $(0-0.1 \mathrm{~Hz})$. This frequency band was selected since TOI correlates with changes in cerebral blood flow in frequencies below $0.1 \mathrm{~Hz}$ [5].

\section{RESULTS}

In figure 2 the results for the projection of the TOI, using the complete set of measurements, are presented. The TOI was decomposed in two signals, one related to the dynamics of $\mathrm{SaO}_{2}$ and another one uncorrelated to it.

In figure 3 the results of the projection of TOI onto the subspaces defined by MABP and $\mathrm{SaO}_{2}$ are shown. The gray line represents the use of a sliding window without the use of a future horizon or bias reduction, the black line represents the results using the proposed method. The upper figure shows the projection onto MABP along $\mathrm{SaO}_{2}$, while the bottom figure shows the projections onto $\mathrm{SaO}_{2}$ along MABP. As can be seen, both projections produce similar results in the first frame (0-18 minutes) but they start to diverge from that point on. Large differences can be observed when comparing the projections onto the subspace defined by the $\mathrm{SaO}_{2}$. In particular, around minute 90 a large discontinuity is depicted. It can be seen that by including the proposed correction this discontinuity is reduced. The larger difference
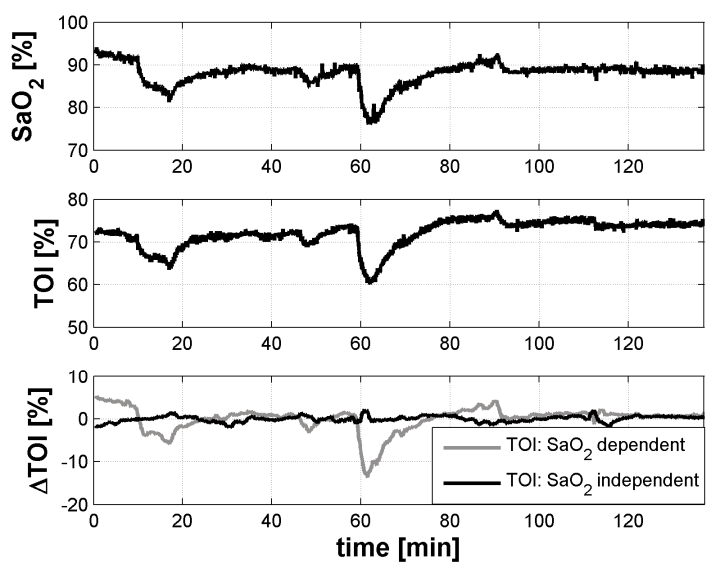

Figure 2. TOI projection onto the subspace defined by $\mathrm{SaO}_{2}$ and the subspace defined by MABP. From top to botton: $\mathrm{SaO}_{2}$ variations, TOI variations, and finally the decomposition of the TOI.

on the projections onto the subspace defined by $\mathrm{SaO}_{2}$ might be explained by the low frequency content of the $\mathrm{SaO}_{2}$ signal, since slow oscillations will produce large changes in the mean value between consecutive frames. Figure 4 shows the results for the proposed methodology and the projections using the complete set of measurements. It can be seen that the projections onto the $\mathrm{SaO}_{2}$ using both methods are similar, while, the projections onto the MABP differ largely. This effect can be attributed to the presence of nonstationarities. Since the relation between TOI and MABP is affected by CA mechanisms that vary in time, when using a large frame that contains nonstationarities, the corresponding projection matrix will represent the subspace defined by the average of the nonstationarities present in the frame. By reducing the frame length, the amount of nonstationarities is reduced, and the subspace represented by the projection matrices will be more related to the local behavior of the signals in that time frame. Therefore, variations in the projections of TOI onto the MABP subspace when using larger and shorter frames are expected.

In table 1 the results for the correlation and coherence values
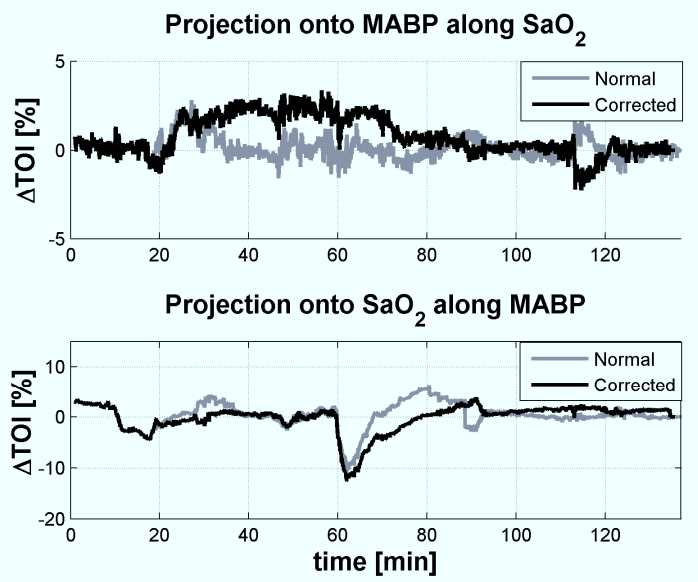

Figure 3. Differences between consecutive projections using a sliding window approach with (black line) and without (gray line) correction for discontinuities. The upper figure represents the projection of the TOI onto the MABP subspace along the $\mathrm{SaO}_{2}$ subspace; while the bottom figure represents the projection of the TOI onto the $\mathrm{SaO}_{2}$ subsnace along the MABP subsnace. 


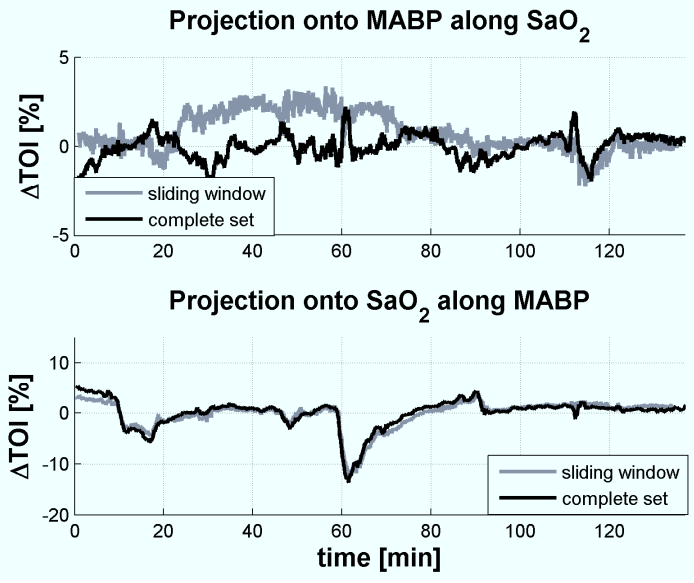

Figure 4. Differences between projections using a complete set of measurements(black line) or the proposed sliding window approach with correction for discontinuities (gray line).

between the TOI, before and after the preprocessing, and $\mathrm{SaO}_{2}$ are shown. The values are presented as median, $25^{\text {th }}$ and $75^{\text {th }}$ percentile. It can be seen that the preprocessing reduced the correlation and coherence values between $\mathrm{SaO}_{2}$ and TOI. A larger reduction is observed in the correlation coefficients, this is due to the fact that the projection matrices tend to reduce the correlation coefficients, and not the coherence. Since an oblique projector is being used, the correlation values are not zero. Coherence values are quite low before preprocessing, this is due to 2 main factors: first of all the coherence between TOI and $\mathrm{SaO}_{2}$ is expected to be higher in low frequencies, by averaging up to $0.1 \mathrm{~Hz}$ the coherence values will be damped by the influence of incoherent high frequency bands close to $0.1 \mathrm{~Hz}$; second, in the analyzed dataset there were not many segments with huge variations in $\mathrm{SaO}_{2}$ which produced lower values of coherence before preprocessing.

\section{CONCLUSION}

In this study we have shown that orthogonal and oblique subspace projection algorithms can be adapted for online monitoring applications. A methodology to reduce the effect of discontinuities in the projections of consecutive segments has been proposed. We have explored its use as a preprocessing step in the CA framework, where the influence of $\mathrm{SaO}_{2}$ on NIRS derived signals has been subtracted.

Under conditions of stationarity the proposed consecutive projections, using a sliding window approach, converge to the projections using the complete set of measurements. However, in the presence of nonstationarities both

Table 1. Correlation and coherence values between $\mathrm{SaO}_{2}$ and TOI before and after preprocessing. Values presented as median $\left(25^{\text {th }}-75^{\text {th }}\right.$ percentile $)$

\begin{tabular}{|l|c|c|}
\cline { 2 - 3 } \multicolumn{1}{c|}{} & $\begin{array}{c}\text { Before } \\
\text { Preprocessing }\end{array}$ & $\begin{array}{c}\text { After } \\
\text { Preprocessing }\end{array}$ \\
\hline \multirow{2}{*}{ Correlation } & 0.38 & 0.20 \\
& $(0.16-0.60)$ & $(0.06-0.38)$ \\
\hline \multirow{2}{*}{ Coherence } & 0.18 & 0.14 \\
& $(0.15-0.23)$ & $(0.13-0.16)$ \\
\hline
\end{tabular}

projections diverge, since the sliding window approach will represent the local behavior of the signals, while the projections using the complete set of measurements will represent an average of the signals behavior in that window. The selection of the window length depends on a trade-off between the slowest dynamics of interest and the reduction of nonstationary effects. Further research to indicate the optimal size of the window using this methodology is needed.

\section{REFERENCES}

[1] Lassen N.A., "Cerebral blood flow and oxygen consumption in man," Physiol. Rev. 1959, 39(2), pp. 183-238.

[2] Soul J.S, Hammer P.E., Tsuji M., Saul J.P., Bassan H., Limperopoulos C., Disalvo D.N., Moore M., Akins P., Ringer S., Volpe J.J., Trachtenberg F., du Plessis A.J. "Fluctuating pressure-passivity is common in the cerebral circulation of sick premature infants". Pediatr. Res. 2007, 61(4), pp. 467-473

[3] Wong F.Y., Leung T.S., Austin T., Wilkinson M., Meek J.H., Wyatt J.S., Walker A.M. "Impaired autoregulation in preterm infants identified by using spatially resolved spectroscopy". Pediatrics, 2008 121(3), pp. e604-e611.

[4] Caicedo A., De Smet D., Naulaers G., Ameye L., Vanderhaegen J., Lemmers P., Van Bel F., Van Huffel S.., "Cerebral tissue oxygenation and regional oxygen saturation can be used to study cerebral autoregulation in prematurely born infants," Pediatr. Res. 2011, 69(6), pp. 548-553.

[5] Wong, F.Y., Nakamura M., Alexiou T., Brodecky V., and Walker A.M. "Tissue oxygenation index measured using spatially resolved spectroscopy correlates with changes in cerebral blood flow in newborn lambs". Intensive Care Med. 2009, 35(8), pp. 1464-1470.

[6] van Bel F., Lemmers P., and Naulaers G., "Monitoring neonatal regional cerebral oxygen saturation in clinical practice: value and pitfalls". Neonatology, 2008, 94(4), pp. 237-244.

[7] Brady K.M., Lee J.K., Kibler K.K, Smielewski P., Czosnyka M., Easley R.B., Koehler R.C., and Shaffner D.H., MD. "Continuous time-domain analysis of cerebrovascular autoregulation using nearinfrared spectroscopy". Stroke 2007, 38(10), pp. 2818-2825.

[8] Hahn G.H., Heiring C., Pryds O., Greisen G. "Applicability of nearinfrared spectroscopy to measure cerebral autoregulation noninvasively in neonates: a validation study in piglets," Pediatr. Res. 2011, 70(2), pp. 166-170.

[9] Caicedo A., Tachtsidis I., Papademetriou M.D. and Van Huffel S. "Decoupling the influence of systemic variables in the peripheral and cerebral haemodynamics during ECMO procedure by means of oblique and orthogonal subspace projections". Proceedings of the 2012 Аnпu. Conf. IEEE EMBS, San Diego, USA, 2012, pp. 61536156.

[10] Behrens R.T., and Scharf L.L. "Signal Processing Applications of Oblique Projection Operators”. IEEE Trans. Signal Process. 1994. 42(6) pp. 1413-1423.

[11] Yanahi H., Takeuchi K., and Takane Y. "Projection matrices, Generalized Inverse matrices, and Singular Value Decomposition". New York: Springer, 2011.

[12] Ingrid Daubechies et al. Ten lectures on wavelets, volume 61. SIAM, 1992. 